\title{
Semantic Processing Ability in Persian-Speaking Alzheimer's Patients
}

Omid Azad ${ }^{1^{*}}$

1. Department of Linguistics, Faculty of Persian Literature and Foreign Languages, University of Allameh Tabataba'i, Tehran, Iran.

Citation: Azad O. Semantic Processing Ability in Persian-Speaking Alzheimer's Patients. Iranian Rehabilitation Journal. 2016; 14(4):211-216. https://doi.org/10.18869/nrip.irj.14.4.211

Article info:

Received: 13 Jul. 2016

Accepted: 02 Nov. 2016

Keywords:

Alzheimer's, Dementia, Executive function,

Comprehension

\section{ABSTRACT}

Objectives: This paper aims to explore whether the Persian-speaking patients of different stages, ranging from mild to moderate, have a deficit in semantic processing by comparing the performance of Alzheimer's patients with that of the healthy individuals.

Methods: The subjects of both the groups of Alzheimer's patients and healthy control were matched for age, the state of monolingual or bilingual, and socio-cultural status. In order to assess the semantic processing ability of the subjects, Pekkala's 2004 model was adopted. According to the model, the subjects were required to produce (say) the name of as many as category members of animals as possible within the time limit of 60 seconds.

Results: The findings showed that while healthy subjects had an intact semantic processing ability, the $\mathrm{AD}$ patients showed weak performance in the five measures of semantic fluency including the number of true linguistic units, the total quantity of words, word size in clusters, the mean cluster size, and the cluster switching.

Discussion: Conclusion: Following the framework of Troyer (1998b), it has been concluded that $\mathrm{AD}$ patients suffer from the semantic processing.

\section{Introduction}

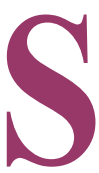

emantic memory, which acts as a pivotal unit of the long-term memory, includes the constant representation of semantic concepts, linguistic units along with their meaning. and transforms our sensory experiences into meaningful units [1]. It can derive the meaning from otherwise meaningless words and linguistic expressions and can make us learn about novel concepts by applying our knowledge learned from past phenomena [2].
Furthermore, appropriate linguistic communication entails us to have sufficient knowledge about our surrounding world, in order to be equipped with satisfactory linguistic knowledge for proper utilization of the linguistic rules, i.e., to possess a healthy semantic memory. Although Alzheimer's disease is considered as one of the most outstanding neurodegenerative diseases, the evidences revealed that the episodic memory is not only negatively affected but also results in the extreme impairment of semantic memory, which is envisaged as the key parameter of the disease [3, 4]. Despite the consensus on the semantic deficit in $\mathrm{AD}$, researchers have

* Corresponding Author:

Omid Azad, PhD

Address: Department of Linguistics, Faculty of Persian Literature and Foreign Languages, University of Allameh Tabataba'i, Tehran, Iran

Tel: +98 (11) 4481789

E-mail: oazad62@yahoo.com 
opposing ideas regarding the nature of semantic memory deficit. While some researchers asserted that deficit in semantic memory originates due to semantic knowledge deficiency $[5,6]$, others insisted that semantic memory deficit arises from disorders in the retrieval of the semantic information $[7,8]$.

Semantic fluency task, also called generative naming task, is most often practiced as a sensitive task by researchers to investigate the semantic processing ability. In fact, it is a multi-factorial task that could be utilized to assess some of the psycho-linguistic components as categorization, whether the semantic ability is preserved or not. Moreover, it can assess the ability of speakers to dissect categories into smallest elements. As a matter of fact, this task could be conducted by utilizing the appropriate performance of working memory, which could play a key role in the transient protection of utterances and their analyses. Alike other cognitive tests, this test aims at divulging and testifying profound mind capabilities. Central executive plays a pivotal role in the whole memory system, considering that it manages a lot of crucial tasks including the rapid investigation of effective, ordered, and focused linguistic elements as well as the flexible transformation of semantic units and sub-units [9]. Furthermore, it should be noted that every item that is processed and retrieved could be most importantly monitored by working memory, whose major role is to block the repetition of retrieved previous words and dissect principles in fluency name test. Moreover, performance in this task may be affected by various nonlinguistic features, including image drawing and the potentiality to produce pictures that arise from vision [5].

Semantic fluency task is regarded as an important diagnostic tool to assess the deficiency of semantic memory in the patients afflicted by Alzheimer's patients [10, 11].
To perform appropriately in the task, the participants are expected to generate words of the members of specific semantic group, such as animals, birds, and fishes, etc. Despite the abundant quantitative researches that have analyzed patients' performance in the semantic fluency tasks $[3,6]$, only a few studies have investigated its qualitative aspects [9]. This study aims at showing whether Persianspeaking Alzheimer's patients show deficits in semantic processing ability. Pekkala's (2004) model was adopted to see what kinds of the qualitative cognitive aspects of semantic fluency are more prone to be damaged [12].

\section{Methods}

\section{Subjects}

Participants were divided into two groups, one as Alzheimer's patients (AD) and another as healthy control group, and were matched according to their age, education, social class, sex, and linguistic category (i.e., monolingual or bilingual). The $\mathrm{AD}$ group included five monolingual patients having no history of tumor, focal neurological sign, alcohol abuse, and neurological or psychiatric disease as severe depression. All the patients were between 65 to 85 years of age and were suffering from $\mathrm{AD}$ for four to eight years. However, the participants should have good reading and writing abilities in order to perform the task. The patients, the members of Iran Alzheimer Association, were under the regular supervision and care services of Association's doctors and psychologists. The control healthy subjects included the participants of Iran Alzheimer Association's sensitive test (test of teaching the elderly healthy people to prevent Alzheimer's disease) group. The severity of dementia in patients was evaluated by utilizing Mini-Mental State Examination (MMSE), which was devised originally by Folstein et al. (1975). The patients securing mark less

Table 1. Demographic information of AD's patients.

\begin{tabular}{cccccc}
\hline Patient & A & B & C & D & E \\
\hline Age & 75 & 76 & 84 & 74 & 82 \\
Sex & Male & Female & Female & Female & Male \\
\hline Occupation & Retired employee & Self-employment & House keeper & Retired teacher & Retired employee \\
\hline Education & High school & High school & High school & MA & Diploma \\
\hline Vernacular & Farsi & Farsi & Farsi & Farsi & Farsi \\
\hline Disease history (years) & 7 & 3 & 8 & 4 & 6 \\
\hline Stage & Early & Early & Moderate & Moderate & Moderate \\
\hline
\end{tabular}


than 8 were excluded and those securing 21 were classified under the mild to moderate stages of dementia [13]. Ultimately, 5 patients were finalized to participate in the task. In Table 1, the thorough demographic parameters of all patients are represented.

\section{Procedure}

The generative naming task utilized in the research is a part of the Boston Diagnostic Aphasia Examination (BDAE). In this test, subjects are required to tell as much as possible names of the related members of different animal categories within the time limit of one minute. The perseveration (the implausible repetition of the previous words), neologism (the creation of novel words), and intrusion (the production of outside category items) were regarded as errors. The Pekkala's (2004) theoretical framework and protocol was adopted for analyzing the collected data based on the subject's performance in 7 parameters. These parameters included 1) the quantity of produced linguistic units, 2) the quantity of semantic clusters (a group composing at least 2 successfully produced words which belong to the similar semantic category, and 3) the mean cluster size. The cluster size was created by applying Troyer's protocol, a linguistic unit designated with a size of cluster marked as 0 , two linguistic units as 1 , three linguistic units as 2 , and so on [14].

\section{Results}

The performance of the AD patients and healthy control participants for each parameter of the fluency task is shown in Table 2.
In order to analyze the performance of the participants in each variable of fluency, an independent t-test was used, which is shown in the Table 1. As per the table, the t-test results showed that the performance of the two groups of Alzheimer's patients and healthy control, based on parameters like true linguistic units, total quantity of words, the linguistic unit clusters, the mean cluster size and the number of switches, were significantly different $(|t|>2.16, P<0.05)$. However, the task performance of both the groups in fluency, including the proportion of words in cluster and number of errors, did not significantly differ $(|t|<2.16, P>0.05)$. In Table 3, the performances of the patients of both the $\mathrm{AD}$ and healthy control groups were compared for each variable of verbal fluency.

Furthermore, to observe whether there is a significant relationship between the cognitive declines of patients and their performance in the semantic fluency task, we used Pearson Correlation Test. The result showed that the performance of the Alzheimer's patients, based on five variables of verbal fluency including the total number of words $(\mathrm{P}=0.002<0.05, \mathrm{r}=0.64)$, the number of correct words $(\mathrm{P}=0<0.05, \mathrm{r}=0.672)$, number of clusters $(\mathrm{P}=0.001<0.05$, $\mathrm{r}=0.63)$, the number of switches $(\mathrm{P}=0.007<0.05, \mathrm{r}=0.595)$ and mean cluster size $(\mathrm{P}=0.015<0.0, \mathrm{r}=0.439)$, is significantly, linearly, strongly, and positively correlated with their performance in MMSE. However, a linear strong negative correlation was found between the patients' performance in the measure of number of words $(\mathrm{P}=0<0.05$, $\mathrm{r}=-0.382$ ) and the result of MMSE. Furthermore, the significant linear correlation was not verified between the performance of the Alzheimer's patients in the measure

Table 2. Performance of subjects in semantic fluency task.

\begin{tabular}{cccccccc}
\hline Subject & A & B & C & D & E & Mean & Control (Mean) \\
\hline TNW & 7 & 7 & 5 & 4 & 7 & 6 & 17 \\
NCW & 6 & 5 & 5 & 3 & 4 & 4.6 & 16.1 \\
NE & 1 & 2 & 0 & 1 & 3 & 1.4 & 0.9 \\
EP & 16.67 & 40 & 0 & 33.33 & 75 & 33 & 5.59 \\
NC & 4 & 3 & 2 & 5 & 2 & 3.2 & 6.6 \\
MCS & 0.75 & 0.67 & 1 & 0 & 0.5 & 0.584 & 1.352 \\
PWC & 50 & 80 & 75 & 0 & 66.7 & 54.34 & 84.92 \\
NS & 4 & 2 & 2 & 5 & 3 & 3.2 & 9.2 \\
\hline
\end{tabular}

Note. TNW=Total Number of Words, NCW=Number of Correct Words, NE=Number of Errors, EP=Error Percentage, NC=Number of Clusters, MCS=Mean Cluster Size, PWC=Proportion of Words in Cluster, NS=Number of Switches. 
Table 3. Performance of $\mathrm{AD}$ patients and healthy control group for the variables of verbal fluency task.

\begin{tabular}{cccc}
\hline Dependent Variable & $|\mathbf{t}|$ & P-value & Result \\
\hline NCW & $5.15>2.16$ & $0<0.05$ & Significantly different \\
\hline TNW & $4.904>2.16$ & $0<0.05$ & Significantly different \\
NC & $3.884>2.16$ & $0.002<0.05$ & Significantly different \\
NS & $5.563>2.16$ & $0<0.05$ & Significantly different \\
MCS & $2.683>2.16$ & $0.019<0.05$ & Significantly different \\
NWC & $0.877<2.16$ & $0.266>0.05$ & Not significantly different \\
NE & $0.877<2.16$ & $0.397>0.05$ & Not significantly different \\
\hline
\end{tabular}

Iranian Rehabilitation Journa

Note: NCW=Number of Correct Words; TNW=Total Number of Words; NC=Number of Clusters; NS=Number of Switching; MCS =Mean Cluster Size; NWC=Number of Words in Cluster; and NE=Number of Errors.

of the proportion of words in clusters and the MMSE result $(\mathrm{P}=0.278>0.05, \mathrm{r}=0.009)$.

\section{Discussion}

The performance of healthy subjects was good in all variables of the task, which showed that healthy control patients had an intact semantic processing ability; whereas the $\mathrm{AD}$ patients had weak performance in five measures of fluency task including the quantity of true linguistic units, the overall linguistic units, the quantity of linguistic units in the semantic clusters, the mean cluster size and cluster switching. Troyer asserted that in the few cases, where patients showed weak performance in cluster size and cluster switching, it could definitely be concluded that the patients might be suffering from semantic processing deficit [15].

The patients in our study showed deficit not only in measures of cluster size and cluster switching but also in other parameters of verbal fluency, including the number of correct words, total number of words, and number of clusters. Hence, in adherence to the viewpoint of Troyer et al, it can be concluded that AD patients in this research suffer from deficits in the semantic processing. In the standpoint of Troyer et al, the temporal lobe is responsible for word clustering while the cognitive variable of the cluster switching is guided by the frontal lobe (ibid). Considering the fact that in Alzheimer's disease, the frontal and temporal lobes are atrophied, it would not have been an unexpected phenomenon to observe the weak performance of our AD patients in the cognitive variables of clustering and switching.
The results of this research are in accordance with those of Hodges and Patterson [6], who found that the semantic memory of Alzheimer's patients compared to those of the healthy control group is impaired. They also proposed that the semantic (verbal) fluency task is the best and most suitable test to assess the semantic processing ability of patients' with AD. Moreover, Troyer et al (1998) conducted the same task on English-speaking patients whose frontal and temporal lobes were damaged and showed that the semantic memory of the patients was also injured. Ultimately, researchers have suggested that in patients with damaged frontal and temporal lobes, the cognitive variables of the number of correct words, switching, clustering, and cluster size are extremely vulnerable.

One of the most important and vulnerable cognitive variables is the cluster size. Furthermore, the finding of the semantic fluency task conducted in this research is partly tantamount to that of Pekkala (2004), in which the performance of the Alzheimer's patients and healthy control subjects did not differ significantly with respect to the variable of the proportion of words in clusters. Unlike Pekkala (2004), the comparative analysis of the patients' performance of the healthy control group to that of $\mathrm{AD}$ group did not show any significantly difference in the measure of number of errors. Moreover, the cluster size in this research has not approximately changed in the experimental group of patients. In our study, the subjects of AD group differed with respect to the variable of cluster size and showed poor performance than those of the healthy control. A significant difference was found in the performance of Alzheimer's patients and the healthy people with regard to the measures of semantic fluency including the cluster size, the overall quantity of correct linguistic 
units, the quantity of words in cluster, switch in semantic clusters, and thorough errors highlighted. The AD patients showed an overall weak performance, whereas the performance of the healthy subjects was good.

Ultimately, as shown in the result section, a strong significant correlation was found between patients' performance in MMSE and their performance in the five variables of the verbal fluency task including the thorough quantity of linguistic units, the quantity of true linguistic units, total semantic clusters, total semantic switches, and the mean cluster size. It was found that the patients' cognitive decline affects their semantic processing ability, i.e., the patient's semantic processing ability decreases with an increase in severity of dementia.

\section{Acknowledgments}

This research did not receive any specific grant from funding agencies in the public, commercial, or not-forprofit sectors. I am grateful to the Chairman and employees of Iran's Alzheimer's Association and Saraye Mehr of Sari who congenially collaborated with me to conduct the research.

\section{Conflict of Interest}

The author declared no conflict of interests.

\section{References}

[1] Squire LR. Memory and brain systems: 1969-2009. The Journal of Neuroscience. 2009; 29(41):12711-6. doi: 10.1523/jneurosci.3575-09.2009

[2] Saumier D, Chertkow H. Semantic memory. Current Neurology and Neuroscience Reports. 2002; 2(6):516-22. doi: $10.1007 /$ s11910-002-0039-9

[3] Martin A, Fedio P. Word production and comprehension in Alzheimer's disease: The breakdown of semantic knowledge. Brain and language. 1983; 19(1):124-41. doi: 10.1016/0093934x(83)90059-7

[4] Hodges JR, Salmon DP, Butters N. Differential impairment of semantic and episodic memory in Alzheimer's and Huntington's diseases: A controlled prospective study. Journal of Neurology, Neurosurgery \& Psychiatry. 1990; 53(12):1089-95. doi: $10.1136 /$ jnnp.53.12.1089

[5] Chertkow H, Bub D. Semantic memory loss in dementia of Alzheimer's type. Brain. 1990; 113(2):397-417. doi: 10.1093/ brain/113.2.397

[6] Hodges JR, Patterson K. Is semantic memory consistently impaired early in the course of Alzheimer's disease? Neuro- anatomical and diagnostic implications. Neuropsychologia. 1995; 33(4):441-59. doi: 10.1016/0028-3932(94)00127-b

[7] Rosen WG. Verbal fluency in aging and dementia. Journal of Clinical and Experimental Neuropsychology. 1980; 2(2):13546. doi: $10.1080 / 01688638008403788$

[8] Tröster AI, Fields JA, Testa JA, Paul RH, Blanco CR, Hames $\mathrm{KA}$, et al. Cortical and subcortical influences on clustering and switching in the performance of verbal fluency tasks. Neuropsychologia. 1998; 36(4):295-304. doi: 10.1016/s00283932(97)00153-x

[9] Troyer AK, Moscovitch M, Winocur G, Leach L. Clustering and switching on verbal fluency tests in Alzheimer's and Parkinson's disease. Journal of the International Neuropsychological Society. 1998; 4(2):137-43. doi: 10.1017/ s1355617798001374

[10] Huff FJ, Corkin S, Growdon JH. Semantic impairment and anomia in Alzheimer's disease. Brain and language. 1986; 28(2):235-49. doi: 10.1016/0093-934x(86)90103-3

[11] Monsch AU, Bondi MW, Butters N, Salmon DP, Katzman R, Thal LJ. Comparisons of verbal fluency tasks in the detection of dementia of the Alzheimer type. Archives of Neurology. 1992; 49(12):1253-8. doi: 10.1001/archneur.1992.00530360051017

[12] Pekkala S. Semantic fluency in mild and moderate Alzheimer's disease. Helsinki: University of Helsinki; 2004.

[13] Folstein MF, Folstein SE, McHugh PR. Mini-mental state: A practical method for grading the cognitive state of patients for the clinician. Journal of Psychiatric Research. 1975; 12(3):18998. PMID: 1202204

[14] Troyer AK. Normative data for clustering and switching on verbal fluency tasks. Journal of Clinical and Experimental Neuropsychology. 2000; 22(3):370-8. doi: 10.1076/13803395(200006)22:3;1-v;ft370

[15] Troyer AK, Moscovitch M, Winocur G, Alexander MP, Stuss D. Clustering and switching on verbal fluency: The effects of focal frontal-and temporal-lobe lesions. Neuropsychologia. 1998; 36(6):499-504. doi: 10.1016/s0028-3932(97)00152-8 
